\title{
¿REDONDILLA O QUADRA? NOTAS SOBRE UNOS VERSOS DEL SIGLO XVII
}

\author{
PABLO MOÍÑO SÁNCHEZ
}

Para José Camões

Cuando, en 1988, Henry Bonneville publica las Poesías humanas del doctor Juan de Salinas, incluye varias composiciones, a menudo atribuidas a otros poetas, cuyo origen todavía es incierto. De entre todas ellas, y a la luz de algunos nuevos hallazgos, me interesa ahora estudiar la redondilla a una melindrosa que cerraba la puerta a las ave marías y después la abría a un fraile procurador de cierto convento:

\author{
¿Qué importa al recato vuestro \\ el que cerréis cada día \\ la puerta al Ave María, \\ si la abrís al Padre nuestro?
}

\footnotetext{
${ }^{1}$ Cito por la edición de Henry Bonneville, Juan de Salinas, Poesías humanas, Madrid: Castalia, 1988, p. 526, que llamaré $P H$ a partir de ahora. Utilizo la abreviatura $C M P B N$ para Edad de Oro-Biblioteca Nacional (dir. Pablo Jauralde), Catálogo de manuscritos de la Biblioteca Nacional con poesía en castellano de los siglos XVI y XVII, 6 vols. y en publicación, Madrid: Arco-Libros, 1993-; CMPHSA designará el Catálogo de los manuscritos poéticos castellanos existentes en la Biblioteca de la Hispanic Society of America (siglos XV, XVI y XVII), por Antonio Rodríguez-Moñino y María Brey Mariño, 3 vols., New York: The Hispanic Society of America, 1965-1966.
} 
Indudablemente, el poema posee las características que de Salinas destacan los estudiosos: juegos de palabras, sencillez expositiva, preferencia por los metros breves, estilo epigramático, intención satírica doble o triple, etc. Bonneville señala que la redondilla aparece en cinco manuscritos. Dos de ellos están íntegramente dedicados a las obras poéticas del doctor: el ms. 6946 de la biblioteca de Antonio Rodríguez Moñino, hoy en la RAE (para Bonneville, $R M)^{2}$ y el add. 17702 del British Museum de Londres (BM)3; a ellos se añaden otros tres donde el poema se atribuye a Villamediana o aparece como anónimo: los llamaremos $H S A^{4}, B N E_{1}^{5}$ y $B N E_{2}^{6}$.

Antes de añadir tres nuevos testimonios castellanos de la redondilla citada, me interesa señalar que Villamediana le disputa a Salinas la autoría de, al menos, otros cinco poemas: las coplas reales «Aunque amor a la razón...» y «Como de tanto dolor...», glosas a las respectivas letras «Volved, Leonisia, a

\footnotetext{
2 Todas las obras del más festivo y galán Apollo, Fénix de España y honor del Parnasso. El Doctor Juan de Salinas, canónigo en la ciudad de Segobia, administrador perpetuo de el hospital de S. Cosme y S. Damián de la ciudad de Seuilla, que murió gloriossamente a los ochenta y dos años de edad, en el del nacimiento de Christo Señor Nuestro el de 1642 [sic], dejando a el mundo un dechado vino de virtud y santidad. Según el estudioso francés, «es quizá el más completo de todos, si bien unas pocas (muy pocas) de las composiciones copiadas parecen de atribución dudosa» (PH, p. 36). Dice Paolo Pintacuda en su estudio del Libro romanzero de canciones, romances y algunas nuebas para passar la siesta a los que para dormir tienen la gana compilato da Alonso de Navarrete: (ms. 263 della Biblioteca Classense di Ravenna), Pisa: ETS, 2005, p. 493, que el códice presenta muchas similitudes con los volúmenes de la BNE ms. 18986 y ms. 10293, dos de los principales cancioneros de Salinas; sin embargo, en dichos manuscritos no aparece nuestra redondilla; tampoco en otros cancioneros importantes del doctor, como los ms. 3948 y 17679 de la BNE (este último, original de sus poemas preparado para la imprenta), entre otros.

3 Obras poéticas del Doctor Joan de Salinas, insigne injenio castellano [sic]. Recogidas por un su aficionado. Es del XVII, anónimo, pero posterior a la muerte de Salinas.

${ }^{4}$ Nueva York, HSA, HC: 380/80. Cf. CMPHSA, ms. CCXVIII: Juan de Tassis..., Chistes sueltos del conde de Villamediana, que no están en lo impreso. Del siglo XVIII. En el f. 214 encontramos los poemas «¿Qué importa el [sic] recato vuestro...»y $\mathrm{y}$ «En la dentadura poca...» atribuidos a Villamediana.

${ }^{5}$ BNE, ms. 3917. Parnaso español. Tomo 6. Cf. CMPBN. Consulta en microfilm: micr. 5454. En los f. 38991 interviene un nuevo copista que recoge varios poemas del doctor Salinas, con su título y explicación correspondientes: «En Fuenmaior, esa villa...» (f. 389-90), «Seáis bien ido, señor...» (f. 390), «Monsiur que al parlamento...» (f. 390-1) y «Ya no más, mi Concepción...» (f. 391); más adelante, anónimas, se copian la décima de Salinas «Ablaba Burgos en cortes...» (f. 393) y la copla de arte menor «Poco importa al onor vuestro...» (f. 396v); esta última es casi idéntica a la redondilla «¿Qué importa al recato vuestro...?»; añade ahora una segunda redondilla y aparece en un folio que incluye varios poemas: una mano que no es la del copista principal ha indicado, al margen de uno de ellos, "Villamediana», pero no parece que la atribución afecte a las composiciones adyacentes.

${ }^{6}$ BNE, ms. 3920. Parnaso español. Tomo 10. Cf. CMPBN. En el f. 281v, sin autor, la redondilla «¿Qué importa el [sic] recato vuestro...?». En los f. 334-7v (los dos últimos folios, a dos columnas) se copia otra composición de Salinas: unos Exercicios de los teatinos que un copista que no es el principal atribuye por error a Góngora y comienzan «Inbidioso que tantos...».
} 
¿Redondilla o quadra? Notas sobre unos versos del siglo XVII

mirar...»y «Obedezco la sentencia...», que se imprimieron en las primeras ediciones de la obra del conde7; la décima «Sacra, real majestad...», que aparece en varios manuscritos de Tassis, pese a que fue compuesta dos años después de su muerte8; la décima "A cinco Isabel tiró...», dedicada a la reina y publicada como de Villamediana en $1886^{9}$; y la redondilla satírica, de estilo muy similar a la que interesa a estas páginas, «Vuestra dentadura poca...»10. Por último, el ms. 4101 de la BNE, no citado por Bonneville y dedicado a la poesía de Villamediana (cf. $C M P B N$ ) incluye un soneto anónimo, atribuido tradicionalmente a Salinas ${ }^{11}$. Eso al menos.

\section{TRES NUEVOS TESTIMONIOS CASTELLANOS}

El ms. 3674 de la BNE (BNE 3$)$, no citado por Bonneville, incluye un pequeño tramo donde se copian varios poemas de Salinas: bajo el título Galería del Santíssimo Sacramento. Salinas, se recoge un total de dieciséis composiciones del doctor en los f. 197-200v, tanto humanas como divinas, entre las cuales se

\footnotetext{
7 En el ms. 4136 de la BNE, Selva de Cupido y Delicioso Jardín de Venus o Poesías amorosas, que a diferentes asumptos, nacidos todos del soberano objeto de su amor, dexó escritos de su mano don Juan de Tassis, conde de Villamediana, sacada de su primitivo orijinal para el excelentísimo señor M.D.S.J., año de 1762 (cf., para su descripción, el CMPBN), se incluyen estas dos glosas, una a continuación de la otra, en las p. 461-9). Los dos poemas, como indica Antonio Carreira en «Nuevos textos y viejas atribuciones en la lírica áurea», en Voz y letra, I/ 2 (1990), 15-142; p. 111, se publicaron en las Obras de Villamediana de 1629 (Çaragoça: Iuan de Lanaja y Quartanet, p. 395-7) y reaparecen en las ediciones posteriores.

${ }^{8}$ En los manuscritos en que se atribuye a Salinas, esta décima - que merecerá un estudio aparte, pues su historia textual es compleja: a menudo aparece mezclada con otras composiciones escritas al mismo asunto, la llegada de Felipe IV y el Conde Duque a Sevilla en 1624 - comienza «Católica majestad...» y es idéntica, en todo lo demás, a la que figura como de Villamediana. Cf. las ediciones de la poesía del conde llevadas a cabo por José Francisco Ruiz Casanova, Poesía impresa completa y Poesía inédita completa, Madrid: Cátedra, 1990 y 1994 respectivamente.

9 En la edición de Poesías del conde, por Emilio Cotarelo y Mori, en El conde de Villamediana, Madrid: Sucesores de Rivadeneyra, 1886, apéndices (p. 233-99). Esta décima aparece en varios manuscritos de Salinas y comienza «Belisa a cinco tiró...»; para Bonneville (PH, p. 469), está dedicada a la duquesa de Osuna.

10 De nuevo en el ms. HC: 380/80 de la HSA, en el mismo f. 214 donde aparece «¿Qué importa el [sic] recato vuestro...», se atribuye a Villamediana esta redondilla, que comienza «En la dentadura poca...» y coincide, con leves variantes, con el poema que Bonneville edita como de Salinas en $\mathrm{PH}$. Por otra parte, existe otro manuscrito de la HSA (ms. B 2492; cf. CMPHSA, ms. CCXI), donde figura como autor de «En la dentadura poca...» Fermín de Sarasa y Arze, f. 86v; parece que la atribución no puede ser cierta, pues lo poco que de Sarasa se conserva pertenece a la década de 1660.

11 Se trata del soneto, anónimo en varios manuscritos, «iOh cargas de un prelado religioso!» (f. $121 \mathrm{v})$, que presenta algunas variantes, una de las cuales mejora notablemente la musicalidad del verso: si en algo disimula, acobardado por el hipermétrico si disimula, de blando es muy sobrado de PH.
} 
encuentra nuestra redondilla12, que aparece sin título y con un error en el primer verso: «¿Qué importa el [sic] recato vuestro...» (f. 198) ${ }^{13}$, lección que comparten, por cierto, dos de los tres manuscritos sí citados por Bonneville: $B N E_{2}$ y HSA. Dejando aparte la posible autenticidad de la atribución con respecto a este poema, lo cierto es que todas las demás composiciones son indudablemente suyas $-\mathrm{o}$ así lo dan a entender numerosos manuscritos de época-, con la honrosa excepción de la redondilla "Vuestra dentadura poca...», ya citada anteriormente a propósito de Villamediana, cuya autoría es más resbaladiza.

También Salinas aparece como responsable de la redondilla en el f. 40v del ms. FD 320 (R 808) de la Biblioteca Nacional de Argentina, en Buenos Aires $(B N A)$, donde el primer verso repite el error: el por al; este códice podría ser copia de alguno de los manuscritos anteriormente citados del doctor ${ }^{14}$.

Por el contrario, el ms. 4147 de la $B N E\left(B N E_{4}\right)$ camina en una dirección muy diferente: en este caso, atribuye la redondilla a don Gabriel de Corral15. Si

12 Este Parnasso cristiano de Pedro de Liévana (cf. CMPBN) contiene, en 202 folios, alrededor de 400 poemas copiados por la misma mano; encuadernado en pergamino con correíllas, su estado de conservación no es bueno: la humedad ha afectado a las tapas y a muchos folios. Todos los poemas humanos aparecen en $\mathrm{PH}$; los de inspiración religiosa se incluyen en otros manuscritos de Salinas. Además de nuestra redondilla (f. 198), aparecen los siguientes: el romance «Ó, si para recibirte...» (f. 197); el romance Desengaño «Porque mi fe i mis desseos...» (f. 197); el romance o Justa al Santísimo Sacramento «Estas sí pueden llamarse...» (f. 197v); la silva de consonantes A una señora que avía de professar en vn convento y tuvo vn mal de garganta, la cual con gargarismos de salmuera estuvo buena; y professó día de san Martín «Cuánto, soror Melchora...» (f. 198); una quintilla A lo mismo: «Porque oi trasciende el jazmín...» (f. 198); un soneto Estando enfermo: «Basta, que me impedís, señor, que os vea...» (f. 198v); dos décimas dedicadas respectivamente a san Bartolomé y a santo Tomé: «A la bodega se entró» y «¿Qué tema es este en que das...» (f. 198v); un Romance frenético, mientras estuvo enfermo en Roma, «Todavía con reliquias...» (f. 199-200, citado en PH como «Con reliquias todavía...»); otra décima, fechada el 7 de mayo y dedicada a las santas «Perpetua y Felicidad...» (f. 200); una décima seguida de cuatro redondillas A la professión de doña Damiana del Espejo, religiosa de Nuestra Señora de los Reies: «En vidrïera preciosa...»; una redondilla A vn fraile mui viejo, mentiroso y falto de dientes («Vuestra dentadura poca...», f. 200v); otra décima en la que habla una llave: «Fuera buena para juez...» (f. 200v) y, por último, dos décimas dedicadas, respectivamente, a tres caballeros y a sus mujeres, «Tres padres de a cuatro cuernos...»y «Las bodas de Arquiticlino» (f. 200v).

${ }^{13}$ No citada por el CMPBN, que por error la considera parte del poema anterior, una quintilla.

14 Poesías inéditas del doctor Juan de Salinas, Sevilla, 1645. Consulta disponible en el catálogo electrónico de la colección Raymond Foulché-Delbosc de la Biblioteca Nacional de Argentina, llevado a cabo por F. A. Marcos Marín, G. Olivetto y V. Zumárraga, con la colaboración de A. L-F Askins (ms. 66; fecha de entrada en el registro, 23-10-1998), última revisión en diciembre de 2001. Según se indica en dicho catálogo, este manuscrito, que perteneció a Manuel Ferry y Colón, fue copiado por Diego de Arroyo y Figueroa y publicado en 1869 por la Sociedad de Bibliófilos Andaluces (lo mismo se decía del ms. 17679 de la BNE).

15 BNE, ms. 4147. [Papeles varios políticos y satíricos sobre acontecimientos y personajes del reinado de Felipe IV, principalmente]. Cf. CMPBN, de donde se toma el título. En el f. 486 se incluye una redondilla, no citada en el CMPBN, atribuida a don Gabriel de Corral; un poco más abajo, se 
¿Redondilla o quadra? Notas sobre unos versos del siglo XVII

bien minoritaria, esta atribución no es única: también se considerará a Corral autor de la redondilla en la Floresta de varia poesía publicada por Adolfo de Castro en el vol. XLII de la BAE ${ }^{16}$.

Recapitulemos. De los ocho testimonios castellanos conocidos, cuatro atribuyen la redondilla a Salinas $\left(R M, B M, B N E_{3}, B N A\right)$, uno a Villamediana (HSA), uno a Corral $\left(B N E_{4}\right)$ y dos permanecen anónimos $\left(B N E_{1}\right.$ y $\left.B N E_{2}\right)$. Por otra parte, cabe señalar que tanto $B N E_{1}$ como $H S A$ y $B N A$ presentan una versión ampliada del poema, que añade una redondilla a modo de respuesta y queda convertida en copla de arte menor ${ }^{17}$.

\section{2. (PERO) UN NUEVO TESTIMONIO PORTUGUÉS}

A la luz de un hallazgo fortuito en la Biblioteca Nacional portuguesa (BNP), el asunto se complica y no solo plantea problemas de autor sino también de idioma original: en el f. 7v del cod. 3106 aparece, entre los poemas de Tomás de Noronha, la siguiente redondilla dedicada

A huã mulher $q$ tinha heça filha a qual desia ser $m^{\text {to }}$ honrrada e a sua porta se fechaua as Aue Marias e a sobredita corria com hum clerigo a quem abriaõ a porta de noite $\mathrm{p}^{\mathrm{a}}$ entrar:

Que importa ao credito uosso

que fecheis todos os dias

as portas as Aue Marias

se abris ao Padre nosso?

copia la redondilla «¿Qué ynporta al recato buestro...», con el título A una muger que zerraba su puerta al Abe María y después se la abría a un frayle y la indicación «es del mismo».

16 Cf. Antonio Carreira, art. cit., p. 115. Desgraciadamente, en la edición de la BAE no se indica la fuente, que podría haber sido el propio ms. 4147; la redondilla no está en otras ediciones de Corral, ni aun en la más moderna: Obras de Gabriel de Corral, introducción, edición y notas por John V. Falconieri, nota preliminar de Lorenzo Rubio González, Valladolid: Servicio de Publicaciones de la Diputación Provincial, 1982.

${ }^{17}$ En la p. 526 de $P H$, Bonneville recuerda: «En $R M$, sigue a ésta otra redondilla que trae la rúbrica Respuesta; y es la siguiente: Abrirla al Ave María / no es por el intento vuestro, / sino porque el Padre nuestro / nos da el pan de cada día». La descripción de BNA a través de su catálogo en red muestra que la segunda redondilla comienza allí del mismo modo. Por su parte, la letra del copista es más complicada en $B N E_{1}$ : una posible lectura de la segunda redondilla, con reservas, sería: Si abre vuestra osadía / con atreuimiento diestro, / será porque el Padre Nuestro / nos da el pan de cada día. 
Como se ve, el poema es prácticamente un calco portugués de la redondilla castellana... o viceversa. Fue publicado por primera vez, con leves variantes, en Poesías inéditas de D. Thomás de Noronha, poeta satírico do sec. XVII, edição revista e annotada por Mendes dos Remédios, Coimbra: França Amado, 1899, a partir de tres códices de la Universidad de Coimbra18. Hasta ese momento, nos explica el autor de dicha edición, tan solo se habían publicado poemas de Noronha en la Fénix Renascida19; los que se añaden ahora son el resultado de una «operação de sanidade moral» (p. XXXIV) consistente en la eliminación de poesías que «não se publicam por serem pornographicas» (p. XXXII). En efecto: Noronha, que sigue las formas estróficas habituales - décimas, redondillas, coplas castellanas, sonetos-, cultiva con desenfado la sátira y la burla, con cierta procacidad que recuerda mucho a la del doctor Salinas.

Tras este primer acercamiento de Mendes dos Remédios, tendrán que pasar muchos años para la aparición de otro trabajo monográfico dedicado a Noronha: será la tese de mestrado de Adelino Duarte Neves, Poemas de D. Tomás de Noronha: edição do manuscrito 49-III-71 da Biblioteca da Ajuda de Lisboa, en 1992, que supone un importantísimo avance en las investigaciones acerca del poeta portugués. Para cada uno de los testimonios del códice 49-III-71 de Ajuda, Neves recoge un inventario de variantes. Vemos, de este modo, que la redondilla "Que importa ao crédito vosso...» aparece en este manuscrito y en otros doce, con variantes nunca demasiado significativas. Posteriormente, otro mestrado, el de Teresa Paula Lopes Alves (1996) ${ }^{20}$, asume el trabajo de Neves, corrige algunos (pocos) errores y añade el testimonio del cod. 3106 de la BNL, que no había sido considerado hasta entonces. Por último, la tesis doctoral de Francisco Topa ofrece un nuevo testimonio de autoría que hemos de desechar por cuestiones cronológicas: el poeta brasileño Gregório de Matos (1633-1696),

18 Variantes comunes a la mayor parte de manuscritos conocidos y que mejoran el tercer verso, hipermétrico en el cod. 3106 de la BNP. A uma mulher acautelada em fechar a porta, mas diziam que andava com o cura: «Que importa ao crédito vosso / fechardes, todos os dias, / a porta às AveMarias, / se a abris ao Padre nosso?».

19 A fenis renascida ou obras poéticas dos melhores engenhos portuguezes..., 5 vols., Lisboa: Miguel Rodrigues, 1716-1728 (los poemas de Noronha, en el tomo V, p. 218-57); digitalizada, es posible su consulta a partir del catálogo en red de la BNP. Los manuscritos de la BGUC son el 321, el 391 y el 392. Durante el siglo XX, como veremos más adelante, Noronha aparecerá en diversas antologías.

20 Teresa Paula Lopes Alves, mestrado em Linguística Portuguesa Histórica (maio 1996, univ. Católica Portuguesa, Faculdade de Filosofia e Letras de Braga). La autora añade otras fuentes impresas donde aparece la redondilla: Vitor Manuel Pires de Aguiar e Silva, Maneirismo e Barroco na Poesia Lírica Portuguesa, Coimbra: Centro de Estudos Românicos, 1971, p. 450; Fernando Ribeiro de Mello, Antología do Humor Português, Lisboa: Afrodite, 1969, p. 98; Maria Lucília Gonçalves Pires, Poetas do Período Barroco, Lisboa: Comunicação, 1985, p. 100; Natália Correia, Antología da Poesia do Período Barroco, Lisboa: Moraes, 1982, p. 51. De nuevo Teresa Paula Lopes Alves publicó en 1997 unas Novas poesias inéditas de D. Tomás de Noroña, Braga: APPACDM, 1997; como es lógico, ya no se incluye la mencionada redondilla. 
responsable de la redondilla para el cod. 552 del Fundo Manizola de la BPE: Poesias lyricas de Gregorio de Mattos ${ }^{21}$. Topa cita, en otro lugar, una nueva versión anónima del poema, sin variantes significativas ${ }^{22}$.

De modo que la lista de manuscritos portugueses se eleva, así, a dieciséis: siete en Coimbra, cinco en Lisboa, tres en Évora y uno en Ajuda. Tan solo en dos de ellos el poema figura como anónimo; todos los demás, salvo uno - evidentemente equivocado-, consideran a Noronha como autor ${ }^{23}$.

Después de este vistazo a los testimonios conocidos, castellanos y portugueses, que mencionan nuestra redondilla $-\mathrm{y}$ que, a buen seguro, no serán los últimos en aparecer-, ya solo cabe estudiar los manuscritos, cuadrar fechas y analizar el posible grado de verdad de las diversas atribuciones. Como vemos, la tradición portuguesa es mucho más amplia (dobla en número a la española) y convergente en Tomás de Noronha, nacido en la villa de Alenquer en fecha incierta, a finales del XVI, y muerto en 165124. De los tres poetas españoles, Juan de Salinas (¿1562?-1643) es el de mayor edad; si bien no estamos completamente seguros de su fecha de nacimiento, podemos concluir que lo separan unos veinticinco años del más joven, Gabriel de Corral (1588-1646), casi coetáneo del conde de Villamediana (1582-1622).

A la vista de estos datos, podemos concluir que existe un intervalo de unos quince años (1607-1622, aproximadamente) entre los cuales la redondilla podría haber sido escrita por cualquiera de ellos. Por desgracia, las alusiones cronológicas - muy pocas - que salpican algunos de los manuscritos conocidos no nos permiten eliminar a ninguno de los cuatro; la más temprana data de

${ }^{21}$ Francisco Topa, Edição crítica da obra poética de Gregório de Matos, vol. I, tomo 2: recensio (2. ${ }^{a}$ parte): ed. del autor, Porto, 1999, p. 289: se señalan, asimismo, muchos de los códices - no todos - ya citados por Neves o Alves. Hay que tener en cuenta algo que no señala Topa: la redondilla aparece justo al final del códice, junto con otros dos poemas; y las dos últimas páginas (f. 183v-4) están copiadas por otra mano y probablemente en otra fecha.

22 Se trata del cod. 542 del Fundo Manizola de la BPE, de letra muy tardía, junto con otras Varias quadras a dives [sic] assumptos (p. 155-6, algunas anónimas). Cf. Francisco Topa, «Duas quadras inéditas de José Basílio da Gama», en la Revista da Faculdade de Letras - Línguas e Literaturas, II série, vol. XV, Porto, Faculdade de Letras, 1998, p. 453-4.

23 Instituto dos Arquivos Nacionais da Torre do Tombo (IANTT), ms. 1818, f. 316; Biblioteca da Ajuda (BA), ms. 49-III-71, f. 327; Biblioteca Geral da Universidade de Coimbra (BGUC), ms. 321, f. 22v; BGUC, ms. 358, p. 87; BGUC, ms. 359, f. 50; BGUC, ms. 362, f. 225; BGUC, ms. 364, p. 120 (anónimo); BGUC, ms. 390, f. 59v; BGUC, ms. 392, f. 279v; Biblioteca Nacional, Colecção Pombalina (BNL CP), cod. 133, f. 286v; Biblioteca Nacional, Fundo Geral (BNL FG), cod. 3106, f. 7v; BNL FG, cod. 8581, f. 6; BNL FG, cod. 8594, f. 33; Biblioteca Pública de Évora (BPE), ms. CXIV d/1-14, f. 196v; BPE, Fundo Manizola (BPE FM), cod. 542 (anónimo), f. 453-4; BPE FM, cod. 552, f. 183v-4 (Gregório de Matos).

24 «Morreo na patria em idade provecta no anno de 1651», dice Diogo Barbosa Machado en el tercer tomo de su Bibliotheca Lusitana..., Lisboa: Ignacio Rodrigues, 1752, p. 746. Lopes Alves aclara en su mestrado (p. 15 nota) que en el ms. BA 49-III-71, p. 143 se dice, seguramente por error, que Noronha muere el 4 de enero de 1664. 
1644, pero en ningún caso se alude a la fecha de composición del poema ${ }^{25}$. Los dos códices dedicados a la poesía de Salinas tampoco son de gran ayuda, pese a que el poeta sevillano es el autor hacia el cual apuntan los testimonios españoles de manera más clara. Como ya vimos al comienzo, si nos guiamos por criterios estilísticos no será difícil asignar la redondilla al doctor Salinas; pero las sátiras de Corral y, sobre todo, Villamediana también fueron célebres en su tiempo, lo que justificaría una atribución más o menos ligera26. Por otro lado, nos encontramos ante cuatro poetas cuyos textos no fueron fijados hasta varios años después de morir: Salinas vio, probablemente incompleto, el original de sus poemas preparado para la imprenta (ms. 17679 de la BNE, cf. supra), pero sus obras no se publicaron hasta 1869; las poesías de Villamediana aparecieron en 1629, siete años después de su asesinato; de Corral no poseemos edición hasta el XIX; la obra de Noronha ha permanecido casi del todo inédita hasta el siglo XX... Con todo, los ejemplos de poemas con atribuciones múltiples son tan abundantes en el barroco que probablemente no tenga sentido buscar las causas de la discordia, más allá de criterios temáticos, en este caso ${ }^{27}$.

El poeta Luis Rosales es autor de un interesante estudio acerca de los trasvases castellanoportugueses en la lírica de los siglos XVI y XVII28; allí se demuestra, a través de las traducciones de Villamediana a Camões o las imitaciones de este a Garcilaso, cómo el contacto entre ambas lenguas era muy acusado $^{29}$. ¿Pudo traducir el conde algún poema de Noronha? ¿De qué modo se trasladó la redondilla del portugués al español o viceversa? ¿Cabría hablar de la traducción voluntaria de un poeta culto o más bien de una transmisión popular o versión ad hoc de alguno de los copistas?

En todo caso, tal vez se podría apuntar que existe una conexión, aunque para encontrarla sean necesarios ciertos malabarismos, entre los dos autores más citados por los manuscritos, Tomás de Noronha y Juan de Salinas. El poeta portugués fue llamado «O Marcial de Alenquer» por su estilo satírico y epigramático ${ }^{30}$; y la denominación recuerda vagamente a otro Alenquer: Diego

\footnotetext{
${ }^{25}$ En su mayor parte, se trata de copias del siglo XVIII o de mediados o finales del XVII. El poema que aparece en $B N E_{4}$ pudo ser escrito en torno a 1644; el de $B N E_{3}$, en 1678; no hay manera de conocer las fechas de $B N E_{1}$ y $B N E_{2}$, que contienen versos de diferentes años; etc.

${ }^{26}$ La transmisión manuscrita de la poesía del conde es especialmente abundante y, en ocasiones, arbitraria, como vimos más arriba. Véase Juan Manuel Rozas, El conde de Villamediana, Madrid: CSIC, 1964, entre otras obras.

27 Cf., por ejemplo, el citado artículo de Antonio Carreira en Voz y Letra.

${ }^{28}$ Luis Rosales, «La poesía cortesana», en El sentimiento del desengaño en la poesía barroca, Madrid: Cultura Hispánica, 1966, p. 127-205.

${ }^{29}$ José Francisco Ruiz Casanova recuerda en su introducción a Poesía impresa... que Tassis tradujo al menos a Camões, Martín Puz y Marino.

30 El sobrenombre de Noronha es bien conocido; Alves, entre otros autores, lo recuerda en su mestrado.
} 
¿Redondilla o quadra? Notas sobre unos versos del siglo XVII

de Silva y Mendoza, duque de Francavila, conde de Salinas y de Ribadeo y marqués de Alenquer, muy habitual, por cierto, en los códices portugueses de los siglos XVII y XVIII. Los copistas designan indistintamente al conde como Conde de Salinas, Salinas, Marqués de Alenquer (con abreviatura en marqués) o simplemente Alenquer. Vemos que, aunque poco probable, por un lado y por otro el desliz es posible: Juan de Salinas $>$ Salinas $>$ conde de Salinas $>$ marqués de Alenquer $>$ M. de Alenquer $>$ Thomás de Noronha; o viceversa.

Tomado todo ello con las reservas necesarias, simplemente se puede concluir que la redondilla «¿Qué importa al recato vuestro...»o «Que importa ao crédito vosso...» es uno más de los habituales trasvases poéticos del siglo XVII, de origen todavía más incierto que al comienzo de este trabajo. 